\title{
Politik und ihre Grenzen in Clausewitz' Denken über den Krieg
}

\author{
Tobias Wille (1D
}

Online publiziert: 19. August 2020

(C) Der Autor 2020

Zusammenfassung Carl von Clausewitz' Denken über den Krieg steht paradigmatisch für ein instrumentelles Verständnis von Gewalt in der Politik. Gewalt ist für Clausewitz ein Mittel, das im Krieg verwendet wird, um politische Zwecke zu erreichen. Seit dem Ende des Ost-West-Konflikts ist jedoch die Ansicht weit verbreitet, dass Clausewitz' Überlegungen keine Gültigkeit mehr besitzen. Gegenwärtige Formen des Krieges seien zwar gewaltsam, aber nicht mehr politisch, weil sie nicht allein von Staaten oder aus einer eng verstandenen Staatsräson heraus geführt werden. Der Einwand missversteht jedoch Clausewitz' Begriff der Politik. Dieser soll im vorliegenden Aufsatz systematisch rekonstruiert werden. Dem zu entwickelnden Interpretationsvorschlag zufolge bezeichnet „Politik“ in Clausewitz' theoretischem System zunächst einmal nur ganz allgemein eine Interaktion von zwei oder mehr Akteuren, die jeweils ihren Willen realisieren wollen, deren Willen sich jedoch nicht vollständig vereinen lassen. Krieg ist für Clausewitz dann solche Politik, die mit gewaltsamen Mitteln betrieben wird. Vor diesem Hintergrund wird argumentiert, dass Clausewitz' Theorie des Krieges einen fruchtbaren Analyserahmen bietet, mit dem sich die Transformationen der politischen Gewalt von den Kabinettskriegen des 18. Jahrhunderts bis zu den ,neuen Kriegen“ unserer Zeit nachvollziehen lassen.

Schlüsselwörter Carl von Clausewitz · Internationale Beziehungen · Konflikt · Neue Kriege · Politische Gewalt

\section{T. Wille}

Saltzman Institute of War and Peace Studies, Columbia University, 420 West 118th Street, New York, NY 10027, USA

Forschungszentrum „Normative Ordnungen“, Goethe-Universität Frankfurt am Main, Theodor-W.-Adorno-Platz 1, 60323 Frankfurt am Main, Deutschland E-Mail: wille@soz.uni-frankfurt.de 


\section{Politics and its Limits in Clausewitz's Thought on War}

Abstract Carl von Clausewitz's thought on war represents in a paradigmatic fashion an instrumental conception of violence in politics. For Clausewitz, violence is a means used in war to achieve political ends. Since the end of the East-West conflict, however, it is widely thought that Clausewitz's ideas are no longer applicable. Current forms of war may be violent, it is said, but they are no longer political because they are not waged by states alone, for the raison d'état narrowly conceived. This objection, however, rests on a misunderstanding of Clausewitz's concept of politics. This article seeks to reconstruct this concept systematically. It offers an interpretation whereby "politics" in Clausewitz's theoretical system denotes an interaction of two or more actors, each of whom wants to realize their respective will but whose wills cannot be fully reconciled. For Clausewitz, war is such politics that is conducted by violent means. Against this background, it is argued that Clausewitz's theory of war provides a fruitful analytical framework to understand the transformations of political violence from the cabinet wars of the 18th century to the "new wars" of our time.

Keywords Carl von Clausewitz · Conflict · International relations · New wars · Political violence

\section{Einleitung}

Carl von Clausewitz' berühmtes Diktum, der Krieg sei die bloße Fortsetzung der Politik mit anderen Mitteln, gilt als paradigmatische Formulierung der Idee, dass Gewalt ein rationales Instrument der Politik sein kann. Ausbuchstabiert in der monumentalen Abhandlung Vom Kriege ${ }^{1}$, an der Clausewitz mehr als ein Jahrzehnt feilte und die er doch aufgrund seines verfrühten Todes im Jahr 1831 nicht vollenden konnte, hatte diese Idee in der zweiten Hälfte des 19. und dann insbesondere im 20. Jahrhundert einen beachtlichen Einfluss auf die Praxis wie auch die Theorie des Krieges. Die deutschen und französischen Befehlshaber im ersten Weltkrieg, die Generäle der Wehrmacht, Revolutionäre von Lenin bis Mao und Giáp sowie die nuklearen Strateg*innen des Kalten Krieges - sie alle haben Clausewitz gelesen und sich an seinen Ideen abgearbeitet. ${ }^{2}$ In den 1990er- und 2000er-Jahren machte sich dann allerdings die Überzeugung breit, dass Clausewitz' Denken über den Krieg einer vergangenen Zeit angehöre und man mit ihm die „neuen Kriege“ nach dem Ende des Ost-West-Konflikts nicht angemessen fassen könne (Kaldor 2000). Es hieß, Clausewitz habe ausgedient. Dieser Vorwurf hat einer Anzahl von Autor*innen Anlass gegeben, das Werk des preußischen Generals erneut einer genauen Lektü-

\footnotetext{
1 Im Folgenden zitiere ich aus der von Hahlweg herausgegebenen 19. Auflage bei Dümmler (von Clausewitz 1980). Um ein leichtes Auffinden der Stellen in anderen Ausgaben zu gewährleisten, gebe ich außerdem Buch und Kapitel an. Das Format der Verweise ist (VK Buch/Kapitel/Seite).

2 Zur Rezeptionsgeschichte von Vom Kriege siehe Bassford (1994), Hahlweg (1980a,b), Heuser (2005), Howard (1984) und Kinross (2008).
} 
re zu unterziehen (Daase und Schindler 2009; Herberg-Rothe 2001; Heuser 2005; Schindler 2020) und die immer noch vorhandene Relevanz seiner Einsichten durch eine Reihe von Studien zu belegen (Daase 1999; Münkler 2002a, 2006; Wassermann 2015). Dabei wurden insbesondere auch Clausewitz' Schriften zum kleinen Krieg aufgearbeitet und für ein breiteres Fachpublikum erschlossen (Daase und Davis 2015; Heuser 2010; Scheipers 2017, 2018). Der vorliegende Aufsatz trägt zu dieser Literatur bei, indem er durch eine systematische Rekonstruktion die tatsächlichen Grenzen der Anwendbarkeit von Clausewitz' Theorie des Krieges vermisst.

Ausgangspunkt meiner Überlegungen ist die Beobachtung, dass die Kritik derer, die Clausewitz nach dem Ende des Kalten Krieges für nicht mehr aktuell hielten, auf sein Verständnis des Krieges als Instrument der Politik zielte (vgl. Daase und Schindler 2009). Der Krieg, so wurde argumentiert, sei keine Fortsetzung der Politik mehr; damit sei auch der gesamte konzeptionelle Rahmen, den Clausewitz entwirft, nicht mehr zu gebrauchen (van Creveld 1998; Kaldor 2000; Keegan 1997). In einer Variante des Arguments wurde Politik mit staatlichem Handeln gleichgesetzt. Weil in den neuen Kriegen nach dem Ende des Ost-West-Konflikts ,private“, das heißt, nichtstaatliche, Akteure eine zentrale Rolle spielen, könnten die neuen Kriege nicht als eine Fortsetzung der Politik gelten. Eine andere Variante des Arguments bezog sich auf die Handlungslogik. Weil die neuen Kriege nicht von traditionellen politischen Interessen, sondern wahlweise von Gier oder Hass motiviert seien, könne man sie nicht mit Clausewitz' Theorie des Krieges fassen. Den unterschiedlichen Einwänden ist gemein, dass sie Clausewitz ein bestimmtes Verständnis von Politik unterstellen, das auf die jeweils zeitgenössischen Erscheinungsformen organisierter Gewalt nicht mehr anwendbar sein soll.

Diese Kritik wird dem differenzierten Verständnis von Politik, das Clausewitz' Theorie zugrunde liegt, jedoch nicht gerecht. Freilich hatte Clausewitz eine bestimmte Erscheinungsform der Politik vor Augen, als er Vom Kriege schrieb. Das zentrale Anliegen seines intellektuellen Wirkens war, die „neuen Kriege“ seiner Zeit, mit denen Napoleon Europa überzogen hatte, einzuordnen und ihre politischen Implikationen für den Staat Preußen, dem er als Soldat diente, zu ergründen. Die Welt, die er beschreibt, ist eine Welt von Staaten, die Nationen einfassen und um das Überleben, aber auch um Ruhm und Ehre kämpfen. Aber seine Theorie des Krieges ist trotzdem mehr als nur eine Theorie der Kriege seiner Zeit. Vielmehr ist sie, wie ich zeigen möchte, eine Theorie des Wandels der politischen Gewalt in der Geschichte. Das wird jedoch erst ersichtlich, wenn man seinen Politikbegriff durch eine gründliche Lektüre seines Werkes systematisch rekonstruiert. Das Ergebnis dieses Unterfangens wird ein Verständnis von Politik als Interaktion von zwei oder mehr Akteuren sein, die jeweils ihren Willen realisieren wollen, deren Willen sich jedoch nicht vollständig vereinen lassen, und die, um ihren Willen durchzusetzen, Gewalt anwenden können. Solange sich Politik in diesen Begriffen beschreiben lässt, solange kann sie auch mit Clausewitz' Theorie verstanden werden. 


\section{Ist der Krieg heute noch eine Fortsetzung der Politik?}

Den Kern von Clausewitz' voll entfalteter Theorie, wie er sie in den letzten Jahren seines Lebens niederschrieb, ${ }^{3}$ bildet die Bestimmung des Krieges als „bloße[r] Fortsetzung der Politik mit anderen Mitteln“ (VK I/1/S. 210). Diese Mittel sind die Mittel des gewaltsamen Kampfes. ${ }^{4}$ Mit seinem viel zitierten, allerdings auch oft missverstandenen ${ }^{5}$ Diktum bringt der preußische General die Einsicht zum Ausdruck, dass der Krieg selbst eine Form der Politik darstellt, die sich von anderen Formen der Politik nur durch die Mittel unterscheidet, mit denen sie betrieben wird. Deshalb schreibt Clausewitz in einer anderen Formulierung auch, der Krieg sei ,nichts als die Fortsetzung des politischen Verkehrs mit der Einmischung anderer Mittel“, wobei aber „dieser politische Verkehr durch den Krieg selbst nicht aufhört, [...] sondern [...] in seinem Wesen fortbesteht" (VK VIII/6B/S. 990-91). ${ }^{6}$ Wollen wir den Krieg verstehen, dürfen wir ihn deshalb nicht in Isolation betrachten. Wir müssen immer auch seine politische Dimension berücksichtigen. Nur so können wir nachvollziehen, wie es zum Einsatz von Gewalt kommt, warum dieser so und nicht anders verläuft und was seine Folgen sind. In der Betrachtung darf ,der Krieg niemals von dem politischen Verkehr getrennt werden“, denn sonst entsteht ein „,sinn- und zweckloses Ding“ (VK VIII/6B/S. 991).

Das bedeutet aber nicht, dass der Krieg nicht auch seine Eigenheiten besäße, die ihn von anderen Formen der Politik unterscheiden. Für Clausewitz folgen diese Eigenheiten aus dem spezifischen Wesen der Gewaltmittel. „Was dem Krieg [...] eigentümlich bleibt“, schreibt er, ,bezieht sich bloß auf die eigentümliche Natur seiner Mittel“ (VK I/1/S. 210). Doch worin besteht die eigentümliche Natur der Gewaltmittel? Für Clausewitz führt der Einsatz von Gewalt zu drei „Wechselwirkungen“, die dem Krieg eine innere Tendenz der Eskalation zum Äußersten verleihen (vgl. Herberg-Rothe 2001, Kap. 2; Münkler 2002b, Kap. 6). Erstens stellt Clausewitz fest, dass es im Krieg, der ja „ein Akt der Gewalt“ ist, „in der Anwendung derselben“ keine Grenzen gibt (VK I/1/S. 194). Es resultiert eine Dynamik, in der beide Seiten wechselseitig zunehmend schonungslos vorgehen, um nicht ins Hintertreffen zu ge-

\footnotetext{
${ }^{3}$ In diesem Aufsatz befasse ich mich mit Clausewitz' Theorie des Krieges, die er in Vom Kriege darstellen wollte, mit deren Ausformulierung er aber aufgrund seines verfrühten Todes nicht zu einem Ende kam. Seine früheren Schriften interessieren mich hier nur insofern, als vor ihrem Hintergrund einige Lücken und Inkonsistenzen im unvollendeten Manuskript von Vom Kriege weniger verwirren. Zu den Entwicklungslinien in Clausewitz' Denken vgl. die klassischen Texte von Kessel (1987) und Rosinski (1935), sowie in der zeitgenössischen Literatur Daase und Schindler (2009), Heuser (2005, Kap. 2), Münkler (2002b, Kap. 5), Scheipers (2018) und Schindler (2020, Kap. 3).

${ }^{4}$ Clausewitz bezeichnet das Mittel des Krieges mit dem Begriff „Gewalt“, wenn er die Akteure einzeln betrachtet (VK I/1/S. 191-92); geht es um deren Interaktion, also die wechselseitige Gewaltanwendung, so spricht er vom „Kampf“ (VK I/2/S. 222, II/1/S. 269-70). Für eine widersprechende, meines Erachtens interessante, aber letztendlich doch unplausible Lesart, der zufolge der Begriff des „Kampfes“ bei Clausewitz keine Gewaltanwendung voraussetzt, vgl. Schindler (2020, S. 13-15).

${ }^{5}$ Die Missverständnisse werden diskutiert und ausgeräumt in Daase und Schindler (2009).

${ }^{6}$ In Korrespondenz aus den späten 1820er-Jahren - also der Zeit, zu der er seine Gedanken zum politischen Wesens des Krieges entwickelte (vgl. Kessel 1987; Schindler 2020, Kap. 3) - verwendet von Clausewitz (1979, S. 496) auch die Formulierung, der Krieg sei ,nichts als die Fortsetzung der politischen Bestrebungen mit veränderten Mitteln“.
} 
raten. Zweitens kann kein anderes Ziel genügen, als den Gegner vollständig wehrlos zu machen, weil beide Seiten befürchten, dass der oder die jeweils andere sonst zu einem späteren Zeitpunkt den oktroyierten Willen nicht mehr akzeptieren und den Kampf wiederaufnehmen könnte. Drittens müssen beide Seiten all ihre Kräfte mobilisieren, weil sich die Willenskraft, mit der die jeweils andere Seite ihren politischen Zweck verfolgt, nicht im Voraus abschätzen lässt. Nimmt man diese drei Punkte zusammen, ist das vorläufige Ergebnis, dass eigentlich jeder Krieg mit vollem Einsatz und bis aufs Letzte geführt werden müsste. ${ }^{7}$

Der wichtigste Grund dafür, dass der Krieg gewöhnlich jedoch nicht zum ÄuBersten eskaliert, liegt für Clausewitz in dessen politischer Natur. ${ }^{8}$ Weil sich die politischen Zwecke unterscheiden, für die Kriege geführt werden, unterscheiden sich auch die Kriege selbst. Nur wenn die Existenz der Beteiligten auf dem Spiel steht, wird bis zum Äußersten gekämpft; geht es um weniger, ist ein geringerer Einsatz zu erwarten (VK VIII/6B/S. 992). Die Unterschiedlichkeit politischer Zwecke erklärt, ,wie ohne inneren Widerspruch es Kriege mit allen Graden von Wichtigkeit und Energie geben kann, von dem Vernichtungskriege hinab bis zur bloßen bewaffneten Beobachtung“ (VK I/1/S. 201). Clausewitz verortet den Krieg also im Spannungsfeld zweier Kräfte. Zum einen hat er, wie oben erläutert, seine eigenen inneren Dynamiken, die ihm eine Tendenz zur Eskalation einschreiben. Zum anderen ist der Krieg als Instrument der Politik von Erwägungen bestimmt, die ihm äußerlich sind. In der Konsequenz erscheint er als „Halbding“ und als „Widerspruch in sich“, der ,als solcher nicht seinen eigenen Gesetzen folgen kann, sondern als Teil eines anderen Ganzen betrachtet werden muss“ (VK VIII/6B/S. 991). Mit seinen internen Dynamiken habe der Krieg zwar seine ganz ,eigene Grammatik“; eine ,eigene Logik" habe er aber nicht, denn seine Logik ist letztlich die Logik der Politik (VK VIII/6B/S. 991).

Genau an diesem Punkt setzten in den 1990er- und 2000er-Jahren die Kritiker*innen an, denen zufolge Clausewitz' Theorie des Krieges die Erscheinungsformen der organisierten Gewalt nach dem Ende des Kalten Krieges nicht erfassen könne und deswegen auch nicht mehr aktuell sei (Daase und Schindler 2009; vgl. auch Herberg-Rothe und Honig 2007; Schuurman 2010). Ihr Einwand war, dass der Krieg keine Fortsetzung der Politik mit anderen Mittel mehr sei. Das Argument wurde in verschiedenen Varianten vorgetragen, von denen ich hier die zwei wichtigsten etwas ausführlicher diskutieren möchte. Die erste Variante fokussiert auf die Akteure: Clausewitz habe eine Theorie des zwischenstaatlichen Krieges vorgelegt. Die „,neuen Kriege“ nach dem Ende des Ost-West-Konflikts, die nicht mehr ausschließlich und vielleicht sogar nicht mehr vorrangig von Staaten geführt werden, könne die Theorie deshalb nicht fassen. Die zweite Variante des Arguments zielt

\footnotetext{
7 Clausewitz (VK I/1/S. 195-196) spricht hier vom „bloßen Begriff“ des Krieges; die damit verbundene Theorie der Wechselwirkungen sei aber ,ein bloßes Büchergesetz [...] und keins für die richtige Welt“.

8 Ein zweiter Grund, warum Kriege nicht immer zum Äußersten eskalieren, liegt für Clausewitz in der Friktion (VK I/1/S. 209-10; vgl. I/4-7/S. 253-64), die in allen militärischen Aktivitäten auftritt und die Kontrahent*innen davon abhält, ihre gesamten militärischen Mittel zu einem einzigen Zeitpunkt und an einem einzigen Ort zum Einsatz zu bringen. In den meisten Interpretationen wird die Friktion als von der Politik unabhängige, mäßigende Kraft verstanden. Wie ich im nächsten Abschnitt zeigen werde, ist sie jedoch enger mit der Politik verwoben, als auf den ersten Blick ersichtlich ist.
} 
hingegen auf die Handlungslogik: Clausewitz' Theorie des Krieges sei auf ,ppolitisches“ Handeln im Sinne einer eng verstandenen Staatsräson beschränkt. Weil die neuen Kriege aber aus anderen Motiven heraus, insbesondere aus Hass oder Gier, geführt würden, sei die Theorie auf sie nicht anwendbar. Die beiden Varianten des Arguments sind miteinander vereinbar und werden oft gemeinsam vorgebracht.

Der wohl bekannteste Vertreter der ersten Variante des Arguments ist van Creveld (1998, S. 188), für den Clausewitz' Diktum, der Krieg sei eine Fortsetzung der Politik, „nicht mehr und nicht weniger“ besagt, ,als daß der Krieg ein Instrument in den Händen des Staates bilde, soweit der Staat zu politischen Zwecken Gewalt einsetzt". ${ }^{9}$ Politik wird so kurzerhand mit dem Handeln von Staaten gleichgesetzt. Nun hat van Creveld erst einmal Recht, wenn er beobachtet, dass Clausewitz sich an den Kriegen seiner Zeit abarbeitet. Clausewitz geht es darum, dem Übergang vom Kabinetts- zum Volkskrieg Sinn abzugewinnen, und beides sind Formen des zwischenstaatlichen Konflikts. Dennoch liegt van Creveld (1997, S. 9, meine Übersetzung) falsch, wenn er schreibt: „nirgendwo in Vom Kriege zieht [Clausewitz] in Betracht, dass Krieg von anderen Arten von politischen Organisationen geführt werden könnte" (was er dann auch selbst bereits im Folgesatz relativiert). Ganz im Gegenteil: Im 3. Kapitel B des VIII. Buches in Vom Kriege zeichnet Clausewitz die Geschichte der Kriegsführung nach, um zu zeigen, dass ,halbgebildete Tataren, Republiken der alten Welt, Lehnsherren und Handelsstädte des Mittelalters, Könige des achtzehnten Jahrhunderts, endlich Fürsten und Völker des neunzehnten Jahrhunderts“ den Krieg jeweils ,auf ihre Weise“, „,mit anderen Zielen und anderen Mitteln“ geführt haben. Dennoch beharrt van Creveld darauf, dass die Anwendbarkeit von Clausewitz' Theorie auf den zwischenstaatlichen Krieg beschränkt sei.

Um diese These zu untermauern, bezieht sich van Creveld (1998, Kap. 2) - wie auch andere Kritiker*innen (z.B. Kaldor 2000, S. 36-37) - auf die Bemerkungen zur „wunderlichen Dreifaltigkeit“ aus dem 1. Kapitel des I. Buchs in Vom Kriege. Dort schreibt Clausewitz, im Krieg herrschten drei Tendenzen: der „Haß und die Feindschaft, die wie ein blinder Naturtrieb anzusehen sind“, das „Spiel der Wahrscheinlichkeiten und des Zufalls, die ihn zu einer freien Seelentätigkeit machen" und die ,untergeordnete [...] Natur eines politischen Werkzeugs, wodurch er dem bloßen Verstand anheimfällt“" (VK I/1/S. 213). Für die Kritiker*innen besagt diese Passage, dass Clausewitz' Theorie systematisch auf staatliche Akteure mit ihrer ,Trinität von Regierung, Heer und Volk“ (van Creveld 1998, S. 74) beschränkt sei. ${ }^{10}$ Zwar erläutert Clausewitz (VK I/1/S. 213) tatsächlich in Bezug auf die Dreifaltigkeit, dass „die erste dieser drei Seiten [...] mehr dem Volke, die zweite mehr dem Feldherrn und seinem Heere, die dritte mehr der Regierung zugewendet" sei. Ganz abgesehen davon, dass Clausewitz an dieser Stelle gar keine trennscharfe Zuordnung vornimmt, bezieht sich diese Aussage jedoch klar nicht auf das allgemeine Wesen des Krieges,

\footnotetext{
9 Ein anderer ist Keegan (1997, S. 21), demzufolge Clausewitz' Diktum ,neben der Existenz von Staaten auch Staatsinteressen und Strategien [voraussetzt], um solche Interessen zu erreichen“. Eine sehr ähnliche Formulierung findet sich auch bei Kaldor (2000, S. 28).

10 Die Fehlinterpretation der Dreifaltigkeit als drei Komponenten des modernen Nationalstaats hat ihren Ursprung allerdings nicht bei van Creveld, sondern bei Summers (1984, S. 26-27), einem bekennenden Clausewitzianer; vgl. Villacres und Bassford (1995, S. 9-10).
} 
sondern die spezifischen Verhältnisse seiner Zeit (vgl. Herberg-Rothe 2007; Villacres und Bassford 1995). Für ihn sind die Tendenzen nämlich, wie er an derselben Stelle auch schreibt, „,von veränderlicher Größe“; die Aufgabe der Theorie ist genau, dass sie sich ,zwischen diesen drei Tendenzen wie zwischen drei Anziehungspunkten schwebend erhalte“ (VK I/1/S. 213). Die Behauptung, Clausewitz' Theorie sei auf den Krieg zwischen souveränen Staaten beschränkt, erweist sich somit bei einer genauen Lektüre als haltlos.

Die zweite Variante des Arguments, dass Clausewitz nicht mehr aktuell sei, bezieht sich wie schon gesagt auf die Handlungslogik, der die Akteure im Krieg folgen. Während des Kalten Krieges hatte sich insbesondere in den USA eine stark rationalistische Lesart von Clausewitz durchgesetzt, die seine Überlegungen dafür nutzbar machen wollte, durch kühle Berechnung einen Nuklearkrieg zu verhindern oder, falls das scheitern sollte, zumindest geschickt führen zu können (Brodie 1973; Osgood 1957; vgl. Echevarria 2007; Heuser 2005, S. 194-203). Doch diese einseitige Interpretation des preußischen Generals als Theoretiker des rationalen politischen Kalküls erwies sich dann nach dem Ende des Ost-West-Konflikts als wenig hilfreich. Weil die neuen Kriege der 1990er-Jahre einer anderen, vermeintlich unpolitischen Logik zu folgen schienen, erklärten die Kritiker*innen Clausewitz' Theorie des Krieges damit insgesamt für überholt. Als vielleicht prominenteste Vertreterin dieser Position ist Kaldor (2000) zu nennen, die Clausewitz heranzieht, um die „alten Kriege“ zu definieren und von den „neuen Kriegen“ abzugrenzen. Sie betont das komplexe Zusammenspiel zweier Handlungsmotive, die Clausewitz' Theorie des Krieges als Fortsetzung rationaler Politik nicht fassen könne: ethnische Identitäten und ökonomische Interessen. Diese beiden Motive spielen, in unterschiedlicher Gewichtung, eine herausragende Rolle in der gesamten Literatur zu den neuen Kriegen. ${ }^{11}$

Kaldor (2000, S. 15) argumentiert nun in Bezug auf das erste Handlungsmotiv, dass die Ziele der neuen Kriege von einer „Politik der Identität“ bestimmt seien und sich dadurch von den „geopolitischen und ideologischen Motiven“ der alten Clausewitz'schen Kriege unterschieden. Auch andere Autor*innen betonen die Bedeutung von Identitätskonflikten. Für van Creveld (1998, S. 189, 212) ist der „politische Krieg“ gar nur eine „moderne Erfindung“, während die meisten Kriege in der Geschichte um die ,nackte Existenz der Gemeinschaft“ und ohne eine rationale Abwägung von Mitteln und Zwecken geführt wurden und auch nun wieder vermehrt geführt werden; ,ein Krieg dieser Sorte“ sei aber „eben nicht die Fortsetzung der Politik mit anderen Mitteln“. Für Keegan (1997, S. 101) „trotzen“ die Konflikte der 1990er-Jahre auf dem Balkan und im Kaukasus ,,ihrem Wesen nach Vermittlungsbemühungen von außen, da sie auf Leidenschaft und Haßgefühlen beruhen, die rationalen Argumenten kein Gehör schenken; sie sind in einer Weise apolitisch, die Clausewitz kaum vorgesehen hat". In Bezug auf das zweite Handlungsmotiv argumentiert Kaldor (2000, S. 19-20), dass die neuen Kriege in globale ökonomische Kreisläufe eingebettet seien, was unter anderem dazu führe, dass die Akteure keine entscheidende Schlacht mehr, sondern das endlose Schwelen des Konflikts beabsichtigen. Auch für andere Analytiker*innen der neuen Kriege ist Clausewitz'

${ }_{11}$ Die relative Bedeutung der beiden Motive und ihr Verhältnis zueinander wurden ausgiebig in der sogenannten Greed-versus-grievance-Debatte diskutiert (Collier und Hoeffler 2004; Fearon und Laitin 2003). 
Theorie des Krieges nicht mehr anwendbar, weil die neuen Kriege keiner politischen, sondern letztendlich einer ökonomischen Logik folgen. Für sie erscheint der Krieg nicht mehr als eine Form der Politik, sondern als „Fortsetzung der Ökonomie mit anderen Mitteln“ (Keen 1998, S. 11, meine Übersetzung). ${ }^{12}$

All diesen Kritiken ist gemein, dass sie Clausewitz' Theorie des Krieges zurückweisen oder zumindest für überholt erklären, weil gegenwärtige Formen der organisierten Gewalt nicht dem Politikbegriff entsprächen, der seiner Theorie zugrunde liegt. Weil die neuen Kriege keine Fortsetzung der Politik mit anderen Mitteln seien, könne Clausewitz uns auch nicht helfen, sie zu verstehen. Sie ziehen also zeitlich dort eine Grenze für die Anwendbarkeit des Clausewitz'schen Denkens, wo Krieg nicht mehr allein von Staaten und nicht mehr exklusiv aus Gründen der eng verstandenen Staatsräson geführt werden. Wie ich im nächsten Abschnitt zeigen werde, missverstehen sie dabei allerdings den Politikbegriff, der Clausewitz' Denken über den Krieg zugrunde liegt. Wenn man die Rolle der Politik in seinem Denken über den Krieg systematisch rekonstruiert, wird aus meiner Sicht deutlich, dass sich die Transformationen der politischen Gewalt auch über das Ende des Kalten Krieges hinaus mit Clausewitz' Theorie analysieren lassen.

\section{Eine systematische Rekonstruktion des Politikbegriffs bei Clausewitz}

Im Folgenden soll nun also geklärt werden, was genau der Begriff der „Politik“ in Clausewitz' Theorie des Krieges bedeutet. An dieser Stelle sind wir allerdings mit einer exegetischen Schwierigkeit konfrontiert: Während den Krieg in all seiner Komplexität und Wandelbarkeit zu erfassen, das zentrale Anliegen seines theoretischen Schaffens war, hat Clausewitz an keiner Stelle in vergleichbarer Ausführlichkeit erläutert, was er mit „Politik“ meint. Seine Bewundrer*innen wie auch seine Kritiker*innen stützen ihre Interpretationen deshalb vorrangig darauf, wie Clausewitz den Begriff der „Politik“ verwendet, wenn er über empirische Sachverhalte schreibt. So kommen sie zu dem Ergebnis, Clausewitz sei ein politischer Realist gewesen, der über eine Welt eigeninteressierter und rational handelnder Staaten geschrieben habe (z. B. van Creveld 1998, S. 102-106; Gat 1989, S. 239-244; Heuser 2005, S. 60-64; Paret 1992, Kap. 12; vgl. Daase und Schindler 2009, S. 719-720). Diese Lesart verkennt jedoch, dass Clausewitz' Theorie des Krieges sowohl mit einem abstrakten, zeitlosen Begriff von Politik operiert (der allerdings nicht vollständig expliziert wird) als auch ihre konkreten historischen Ausprägungen betrachtet. Der Fehler der Interpret*innen liegt darin, dass sie eine konkrete historische Form der Politik mit dem allgemeinen Begriff der Politik verwechseln, der systematisch die Grenzen der

\footnotetext{
12 Münkler, der sich intensiv mit der Privatisierung der Gewaltanwendung in den neuen Kriegen beschäftigt hat (2002a, 2006), kommt zu einem differenzierteren Urteil. Clausewitz' allgemeine Theorie des Krieges als Akt der Gewalt, mit der ich mich im nächsten Abschnitt befassen werde, scheint Münkler (2007, S. 229-230) auch heute noch Gültigkeit zu besitzen. Dennoch hält er das Diktum vom Krieg als Fortsetzung der Politik für obsolet, weil die neuen Kriege nach dem Ost-West-Konflikt seiner Ansicht nach keiner politischen Logik mehr folgen. Wie ich zeigen werde, hat Clausewitz jedoch einen so weiten Politikbegriff, dass dieser problemlos auch die gewaltsamen Konflikte unserer Zeit fassen kann.
} 
Anwendbarkeit der Clausewitz'schen Theorie demarkiert. Es muss im Folgenden also darum gehen, diesen abstrakten Begriff der Politik zu rekonstruieren.

Wie wir gesehen haben, charakterisiert Clausewitz den Krieg allgemein als eine Form der Politik, die sich durch den Einsatz von Gewalt auszeichnet. Es ist nur die ,eigentümliche Natur“ der Gewaltmittel, die den Krieg von allen anderen Formen von Politik unterscheidet (VK I/1/S. 210). Wenn wir diese Bestimmung ernst nehmen, dann können wir auf das allgemeine Wesen der Politik schließen, indem wir von den allgemeinen Aussagen Clausewitz dazu, was den Krieg ausmacht, den Einsatz der Gewaltmittel abziehen. Genau das soll mein Vorgehen in diesem Abschnitt sein. Um die Schwierigkeit zu überwinden, dass nicht an allen Stellen in Vom Kriege klar ist, ob Clausewitz über das allgemeine Wesen des Krieges schreibt oder über eine seiner spezifischen historischen Formen, orientiere ich mich vor allem an der allgemeinen Bestimmung des Krieges aus dem 1. Kapitel des I. Buches von Vom Kriege, einem Abschnitt des Werkes, den Clausewitz vor seinem Tod gründlich überarbeitet hatte und mit dem er zufrieden war. ${ }^{13}$ Auf das Verhältnis von allgemeiner Theorie und konkreter Geschichte in Clausewitz' Denken werde ich dann im nächsten Abschnitt noch einmal zurückkommen.

Clausewitz beginnt seine Abhandlung Vom Kriege mit einer vorläufigen Definition des Gegenstandes: Der Krieg sei ,ein Akt der Gewalt, um den Gegner zu Erfüllung unseres Willens zu zwingen“ (VK I/1/S. 191). Schon hier wird also die Gewalt als Mittel des Krieges eingeführt und in Relation zu einem Zweck gesetzt, nämlich „dem Feinde unseren Willen aufzudringen“ (VK I/1/S. 192). ${ }^{14} \mathrm{Im}$ Folgenden erläutert Clausewitz dann, wie dieser ,politische Zweck des Krieges“ (VK I/1/S. 200) ${ }^{15}$ der internen Tendenz des Krieges zu Eskalation teilweise entgegenwirkt. Denn je nachdem, was die Beteiligten wollen und wie sehr sie es wollen, werden sie auch den Einsatz ihrer Kräfte bemessen. Sind die durch den Gehalt des Willens bestimmten Zwecke beschränkt, so ist es auch das Aufgebot der Mittel. Betrachten wir Clausewitz' Theorie systematisch, scheint er mit dem Begriff „Politik“ also, wenn er ihn abstrakt gebraucht, zunächst einmal Folgendes zu meinen: Politik ist (gewaltloses oder gewaltsames) Handeln, um den eigenen Willen gegen den Willen eines anderen durchzusetzen. Clausewitz (VK II/3/S. 303) selbst macht dieses von seinen anderen Begriffen implizierte Verständnis von Politik beinahe explizit, wenn er - allerdings über einen etwas verworrenen Absatz hinweg verteilt - schreibt, dass sowohl die Politik als auch der Krieg „ein Konflikt menschlicher Interessen“ seien, der Krieg

\footnotetext{
${ }^{13}$ Darüber, wie weit Clausewitz mit einer von ihm 1927 in einer Notiz angekündigten Überarbeitung des Manuskripts gekommen ist, gehen die Meinungen auseinander (Gat 1989, S. 255-263; Kessel 1987; Rosinski 1935). Einigkeit besteht aber, dass das 1. Kapitel des I. Buches bei seinem verfrühten Tod weit gediehen war.

${ }^{14}$ Clausewitz spricht in diesem Zusammenhang auch vom unmittelbaren Ziel der kriegerischen Handlung, den „Feind wehrlos zu machen“ (VK I/1/S. 192), damit er sich dem Willen unterwirft. So ergibt sich dann eine dreifache Unterscheidung zwischen dem Mittel der Gewalt, dem militärischen Ziel der Niederwerfung und dem extern gegebenen politischen Zweck; vgl. hierzu Beckmann (2011), Daase (2007), Jäger und Beckmann (2011).

15 Im gesamten Werk Vom Kriege werden in Bezug auf den Krieg als Ganzes die Begriffe „Zweck“ und ,politischer Zweck“ synonym verwendet. Von einem anderen, nicht politischen Zweck des Krieges ist an keiner Stelle die Rede.
} 
sich jedoch dadurch auszeichne, dass sich in ihm der Konflikt „blutig löst, und nur darin ist er von den anderen [Konfliktformen] verschieden“.

Worin die menschlichen Interessen bestehen, die in aller Politik und damit auch im Krieg konfligieren, ist durch Clausewitz' Theorie nicht bestimmt. Die Akteure haben keine notwendigen Zwecke, die etwa durch die menschliche Natur oder die anarchische Struktur des internationalen Systems gegeben wären. Vielmehr setzen sich die Akteure die Zwecke selbst und können sie zu einem späteren Punkt auch wieder verwerfen (z.B. VK I/2/S. 220-221, III/18/S. 414, VIII/5/S. 985). Kessel (1987, S. 137-138) hat allerdings herausgearbeitet, dass Clausewitz mit dem Begriff der „Politik“ nicht nur das von mir hier rekonstruierte ,subjektive Element“ des Gehalts eines Willens bezeichnet, sondern mancherorts in Vom Kriege auch das „objektive Element“ der allgemeinen politischen Verhältnisse einer Zeit (ähnlich auch Aron 1980, S. 626-627). Clausewitz sieht also, ,daß der Krieg und die Gestalt, welche man ihm gibt, hervorgeht aus den augenblicklich [vorherrschenden] Ideen, Gefühlen und Verhältnissen“ (VK VIII/2/S. 954, von mir berichtigter Druckfehler). Diese objektiven politischen Verhältnisse wirken sich fraglos auf die subjektive „politische Willensbildung“ aus, die aber trotzdem ,zu allen Zeiten und unter allen möglichen Voraussetzungen immer die Freiheit hat, sich so oder so zu entscheiden“" (Kessel 1987, S. 138). Als historisch situierte Subjekte setzen sich die Akteure ihre Zwecke also selbst, tun dies aber vor dem Hintergrund der Verhältnisse ihrer Zeit. ${ }^{16}$ Um begriffliche Verwirrung zu vermeiden, werde ich im Folgenden jedoch nur das subjektive Element als „Politik“ bezeichnen, das objektive Element hingegen als „politische Verhältnisse“.

Dass sich die Akteure in der Politik frei ihre Zwecke setzen, heißt jedoch noch nicht, dass sie sie auch immer realisieren können. Weil die Willen zumindest teilweise gegeneinander gerichtet sind, schränken sich die Akteure wechselseitig in ihrer Freiheit ein. Für Clausewitz (VK I/1/S. 194-95) ist deshalb „der Krieg nicht das Wirken einer lebendigen Kraft auf eine tote Masse“, sondern „,der Stoß zweier lebendiger Kräfte gegeneinander"; wenn aber beide Seiten versuchen, sich gegenseitig mit Gewalt ihren jeweiligen Willen aufzudrängen, so ,bin ich nicht mehr Herr meiner, sondern [der Gegner] gibt mir das Gesetz, wie ich es ihm gebe“. Vollrath (1984) hat in einem Aufsatz zu Clausewitz' Handlungstheorie versucht, diese Verschränkung des Handelns im Krieg mit dem Begriff des „Gegenhandelns“ zu fassen (vgl. auch Herberg-Rothe 2001, S. 160-174). „Im Handeln“, schreibt Vollrath (1984, S. 57), ,trifft alles Handeln auf anderes Handeln und was aus diesem Zusammenhandeln hervorgeht oder hervorkommt, das ist auch dadurch bestimmt, daß Menschen es nicht gemeinsam gewollt und getan haben, sondern daß ein Handeln sich gegen ein anderes gerichtet hat“. Zwar ist für Vollrath (1984, S. 56) ,nicht alles Gegenhandeln [...] kriegerischer Art, aber im Krieg ist alles Handeln von der Art des Gegenhandelns“. In unserer systematischen Rekonstruktion der Theorie Clau-

\footnotetext{
16 Indirekt beschränken auch die verfügbaren Mittel, welche Zwecke sich die Akteure setzen können, denn „,der politische Zweck ist [...] kein despotischer Gesetzgeber, er muss sich der Natur des Mittels fügen und wird dadurch oft ganz verändert“ (VK I/1/S. 210). Das Bindeglied ist hier die politische Klugheit, denn freilich gibt es den Fall, in dem ,,die Politik sich von gewissen politischen Mitteln und Maßregeln eine falsche, ihrer Natur nicht entsprechende Wirkung verspricht“, nur wird sie dann ,mit ihren Bestimmungen einen schädlichen Einfluss auf den Krieg haben“(VK VIII/6B/S. 995).
} 
sewitz' können wir die Begriffe jedoch noch ein wenig genauer fassen. Denn die Unvereinbarkeit der Willen ist nicht notwendig an die Gewaltanwendung gebunden. Sie ist deshalb nicht nur ein Charakteristikum des Krieges, sondern jeder Politik. ${ }^{17}$ Politik ist das gegeneinander Handeln mehrerer Akteure, deren Willen nicht vollständig vereinbar sind und die sich somit in der Realisierung ihrer Willen zumindest teilweise wechselseitig einschränken.

Doch nicht nur die Zwecke der jeweils anderen schränken die Akteure in der Realisierung ihres Willens ein. Hinzu kommt, dass Politik eine Praxis ist, die sich in Raum und Zeit entfaltet. Um diese weltliche Situiertheit mit Hinblick auf den Krieg zu fassen, führt Clausewitz (VK I/7/S. 262) den Begriff der „Friktion“ ein, die für ihn „dem ziemlich allgemein entspricht, was den wirklichen Krieg von dem auf dem Papier unterscheidet“. Will man den Krieg nämlich nicht nur abstrakt denken, sondern tatsächlich führen, ist man mit einer ganzen Reihe von praktischen Schwierigkeiten konfrontiert. „Das Handeln im Kriege“, schreibt Clausewitz (VK I/7/S. 263), ,ist eine Bewegung im erschwerenden Mittel“; es gleicht dem Gehen im Wasser. Selbst die einfachste Bewegung erfordert die äußerste Anstrengung und dennoch kann man sie nicht präzise ausführen. Clausewitz (VK I/7/S. 262) verdeutlicht den Sachverhalt am Beispiel des Wetters: So können etwa Nebel und Regen verhindern, „daß der Feind zu gehöriger Zeit entdeckt wird, daß ein Geschütz zur rechten Zeit schießt, daß eine Meldung den kommandierenden Offizier findet“" oder auch, ,daß ein Bataillon ankommt, daß ein anderes zur rechten Zeit kommt, weil es statt drei vielleicht acht Stunden marschieren mußte“. In Vom Kriege beschränkt Clausewitz seine Diskussion der Friktion auf militärische Angelegenheiten, denn er will erklären, warum nicht alle Kriege zum Äußersten eskalieren. Doch offensichtlich ist auch gewaltloses politisches Handeln, weil es ebenfalls in der Welt stattfindet, der Friktion ausgesetzt. Man denke als Beispiel etwa an die praktischen Schwierigkeiten, mit denen jene konfrontiert sind, die einen Wahlkampf oder einen Generalstreik organisieren wollen.

Nun erscheinen all die praktischen Probleme der Friktion - das „Heer der kleinen Schwierigkeiten" (VK I/7/S. 263), mit denen sich die an einem Krieg Beteiligten herumschlagen müssen - auf den ersten Blick ganz unpolitisch. Sie folgen nicht aus dem Aufeinanderprallen divergierender Willen, sondern betreffen ganz offensichtlich auch einseitiges Handeln. Selbst wenn es auf dem Planeten nur eine einzige Armee gäbe, so wäre auch diese in all ihren Bewegungen der Friktion ausgesetzt. Vollrath (1984, S. 58-60) hat jedoch herausgearbeitet, dass bei Clausewitz der Begriff der Friktion enger mit dem der Politik verbunden ist, als es auf den ersten Blick erscheint. Denn nur, weil die Beteiligten gegeneinander handeln, macht es einen Unterschied, ob das Bataillon rechtzeitig Stellung bezogen hat und der kommandierende Offizier über die Lage informiert wurde. Wäre der Krieg nicht politisch, könnten die Beteiligten sich Zeit lassen und würden einfach etwas später an ihr Ziel gelangen. So gilt aber, dass der Akteur, der die Friktion geschickter überwindet, auch die besseren Chancen hat, seinen Willen gegen den Willen anderer Akteure durchzusetzen.

\footnotetext{
${ }^{17}$ In seinen allgemeinen Ausführungen zur Handlungstheorie, in denen er sich insbesondere auf Arendt stützt, scheint Vollrath (1984, S. 56-58) in diese Richtung zu argumentieren. Er versäumt es dann aber, seine Einsichten zum Gegenhandeln konsequent auf den Politikbegriff bei Clausewitz zu beziehen.
} 
Friktion steht bei Clausewitz also für nichts anderes als die konkreten empirischen Rahmenbedingungen des politischen Handelns.

Weil er eine situierte Praxis ist, erfordert der Krieg auch eine ganz bestimmte Art des praktischen Wissens. Denn die Friktion entzieht sich einer vollständigen Beschreibung; ,man wird sie [...] theoretisch nie ganz kennenlernen“ (VK I/7/S. 263). Ähnlich verhält es sich auch mit den ,geistigen Kräften und Wirkungen“ oder, wie wir heute sagen würden, Emotionen (VK II/2/S. 285-88). Insbesondere die Gefühle der Angst und des Mutes sind als Reaktion auf die allgegenwärtige Gefahr ein wichtiges Element des Krieges. Und, wie auch die Friktion, widersetzen sie sich all den ,verwerfliche[n]“ Theorieversuchen, die sie in feste Regeln fassen wollen (VK II/2/S. 283). Weil eine vollständige Theorie nicht möglich ist, aus der sich für jede Situation geeignete Handlungsmaximen deduzieren ließen, sind alle Beteiligten am Krieg auf ihre Urteilskraft zurückgeworfen. Es ist kein vollständiges theoretisches Wissen, sondern der „,Takt des Urteils“, durch den ,der kriegserfahrene Offizier bei großen und kleinen Vorfällen, man möchte sagen bei jedem Pulsschlag des Krieges, immer passend entscheiden und bestimmen [wird]“ (VK I/7/S. 264). Clausewitz macht klar, dass grundsätzlich in ,,allen anderen Künsten und Tätigkeiten des Lebens“, wozu man dann sicherlich auch die gewaltlose Politik zu zählen hat, nur verinnerlichtes Wissen wirklich nützlich ist; im Krieg fällt dieser Sachverhalt jedoch aufgrund der ,ewig wechselnden Gestalt der Dinge“ besonders ins Gewicht (VK II/2/S. 299). Weil sie in Raum und Zeit stattfindet, ist alle Politik den Widerständen der Friktion ausgesetzt, doch durch die Gewaltanwendung und die mit ihr einhergehenden Unwägbarkeiten kommt ihr im Krieg eine besondere Bedeutung zu.

Auf den ersten Blick erscheint das gegeneinander gerichtete Handeln in der Politik als symmetrische Interaktion. Clausewitz arbeitet jedoch heraus, dass im Krieg die politischen Zwecke der Beteiligten oft asymmetrisch aufeinander bezogen sind. Denn während jeder Angriff der Realisierung eines positiv bestimmten Zweckes dient, muss sich die Verteidigung erst einmal damit zufriedengeben, den Status quo zu erhalten (VK VI/1/S. 614). ${ }^{18}$ In ihrer extremsten Form als ,,reiner Widerstand“ ist die Verteidigung sogar ganz „Kampf ohne eine positive Absicht“ (VK I/2/S. 220). Trotzdem ist der Widerstand keine ,absolute Passivität“, denn ,ein bloßes Leiden wäre kein Kampf mehr“; vielmehr ist er ,eine Tätigkeit, und durch sie sollen so viele von des Feindes Kräften zerstört werden, daß er seine Absicht aufgeben muss“ (VK I/2/S. 220). ${ }^{19}$ Nur vor diesem Hintergrund lässt sich Clausewitz' Aussage verstehen, nicht etwa der Angriff, sondern die Verteidigung sei ,die stärkere Form des

\footnotetext{
18 Allerdings betont Clausewitz (VK VI/1/S. 615, VI/5/S. 633) auch, dass man sich der Verteidigung mit ihrem negativen Ziel nur in einer Position der Schwäche bedienen muss. Wendet sich das Blatt und man gelangt in eine Position der Stärke, so kann man sich ein positives Ziel setzen und zum Angriff übergehen.

19 Solch eine aktive Verteidigung wird durch eine begriffliche Innovation denkbar, die Clausewitz bereits in seiner Bekenntnisdenkschrift von 1812 (von Clausewitz 1966) vollzogen hat und die die Grundlage seiner Überlegungen zum Verhältnis von Angriff und Verteidigung in Vom Kriege bildet (vgl. Daase 2007). Auch wenn sich eine Seite politisch in der Verteidigung befindet, was nichts anderes bedeutet, als dass „eine Nation für ihre Erhaltung, nicht für Eroberung streite[t]“ (von Clausewitz 1966, S. 742), so kann sie doch auf der Ebene der Strategie offensiv handeln, indem sie den Angreifenden entgegenrückt, oder auf der Ebene der Taktik, indem sie gegen die aufgestellten Angreifenden losschlägt.
} 
Krieges“ (VK VI/8/S. 649; vgl. auch VI/2/S. 614-17). ${ }^{20}$ Weil immer gilt, dass ,ein großer Zweck mehr Kraftaufwand erfordert als ein kleiner", ist der Angriff, der etwas positiv Bestimmtes erreichen möchte, gegenüber der Verteidigung mit ihrem negativ bestimmten Zweck grundsätzlich im Nachteil (VK I/2/S. 220). Zu diesem ganz allgemeinen Vorteil aller Verteidigung, die sich schon aus ihrem Begriff ergibt, kommen für Clausewitz dann auch noch der ganz konkrete „Beistand der örtlichen Lage“, den die Verteidiger*innen gewöhnlich genießen (VK VI/2/S. 614). ${ }^{21}$

Das asymmetrische Verhältnis zwischen dem positiven Zweck eines politischen Vorhabens und dem negativen Zweck des Widerstands ist, wenn man Clausewitz' Theorie systematisch rekonstruiert, vom Einsatz gewaltsamer Mittel unabhängig. Wie er selbst klarstellt, besteht der ,aus Begriff und Zweck sich ergebende Vorteil der Verteidigung“ nicht nur im Krieg; er liegt vielmehr ,in der Natur aller Verteidigung“ (VK VI/1/S. 614). Lediglich der konkrete „Beistand der örtlichen Lage“, der den allgemeinen Vorteil der Verteidigung noch weiter verstärkt, ergibt sich ,aus der Natur des Krieges" selbst (VK VI/1/S. 614). Wenn der allgemeine Vorteil der Verteidigung aber nicht an den gewaltsamen Mitteln hängt, die dem Krieg eigen sind, so muss er auch für die Politik insgesamt gelten. Die paradigmatische Konstellation nicht nur des Krieges, sondern jeder Politik besteht in Clausewitz' Denken also genau darin, dass ein Akteur versucht, seinen Willen durchzusetzen, während sich ein anderer diesem Willen widersetzt. Vor einigen Jahren hat Caygill (2013) aus diesen Überlegungen eine allgemeine Theorie des politischen Widerstands entwickelt. Seiner Ansicht nach nimmt die Idee von der Überlegenheit der Verteidigung in Clausewitz' Denken solch eine zentrale Rolle ein, dass sein großes Werk Vom Kriege eigentlich den Titel Vom Widerstand tragen müsste (Caygill 2013, S. 15).

Durch eine systematische Rekonstruktion seiner Begriffe habe ich in diesem Abschnitt gezeigt, dass für Clausewitz Politik ihrem Wesen nach das Gegeneinanderhandeln von Akteuren ist, die jeweils ihren Willen realisieren wollen, deren Willen sich jedoch nicht vollständig vereinen lassen. Als Krieg bezeichnet Clausewitz dabei solches politische Handeln, das mit dem Mittel der Gewalt ausgeführt wird. Durch sie weist der Krieg bestimmte Eigenheiten auf, die ihn von der gewaltfreien Politik unterscheiden. Wie wir bereits im vorhergegangenen Abschnitt gesehen haben, folgt in Clausewitz' Theorie aus dem Wesen der Gewaltmittel eine inhärente Tendenz des Krieges zu eskalieren. In diesem Abschnitt kam die Erkenntnis hinzu, dass die Gewaltanwendung das Problem der Friktion, mit dem grundsätzlich jedes politische Handeln konfrontiert ist, verschärft und dass sie denen, die sich im Krieg verteidigen, bestimmte zusätzliche Vorteile verschafft, die über die allgemeinen Vorzüge der

\footnotetext{
${ }^{20}$ Genau die entgegengesetzte Behauptung - also dass der Angriff die überlegene Form der Kriegsführung sei - wurde Clausewitz immer wieder in den Mund gelegt und hat, insbesondere im frühen 20. Jahrhundert, die Rezeption seines Werkes maßgeblich geprägt (vgl. Hahlweg 1980a; Heuser 2005, Kap. 5; Howard 1984). In der zeitgenössischen Literatur zu Clausewitz hat es sich vor allem Sumida (2008) zur Aufgabe gemacht, dieses Missverständnis auszuräumen.

${ }^{21}$ Eine ausführliche Diskussion der empirischen Faktoren, die die Verteidigung begünstigen, präsentiert Clausewitz im VI. Buch von Vom Kriege. Heute befasst sich in der Politikwissenschaft eine ganze Literatur mit der empirischen Balance zwischen militärischen Mitteln des Angriffs und der Verteidigung (vgl. Brown et al. 2004). Levy (1984, S. 220) sieht in Clausewitz einen Vordenker dieser Literatur, weil er als einer der ersten „Hypothesen“ zum Verhältnis von Angriff und Verteidigung formuliert hat.
} 
Verteidigung hinausgehen. Gemeinsam machen diese Eigenheiten die „Grammatik“ (VK VIII/6B/S. 991) des Krieges aus, die ihn gewissermaßen als eigenständigen Dialekt der Politik auszeichnet.

\section{Die historischen Transformationen der politischen Gewalt}

Die allgemeine Bestimmung des Krieges als Form des politischen Handelns, das mit gewaltsamen Mitteln ausgeführt wird, ist für Clausewitz keineswegs freischwebende Theorie. Er hat sie vielmehr in der intensiven Auseinandersetzung mit den politischen und militärischen Ereignissen seiner Zeit und in der Absicht ausgearbeitet, die historischen Transformationen der politischen Gewalt besser zu verstehen. Das zentrale theoretische Problem, an dem er sich in den letzten Jahren seines Lebens abarbeitete, war, wie sich die historisch so unterschiedlichen Erscheinungsformen des Krieges unter einen Begriff bringen lassen (Aron 1980, S. 92-102; Schindler 2020, Kap. 3). Den Schlüssel zur Antwort sah er in der Einsicht, dass der Krieg nicht losgelöst von den politischen Verhältnissen seiner Zeit betrachtet werden kann. Nur wenn man ihn ,unter allen Umständen als kein selbständiges Ding, sondern als ein politisches Instrument“ denkt, kann man Clausewitz' Ansicht nach nämlich vermeiden „mit der sämtlichen Kriegsgeschichte in Widerspruch zu geraten“ (VK I/1/S. 212; vgl. auch von Clausewitz 1979, S. 496). Die allgemeine Einsicht in das Verhältnis von Politik und Krieg hilft zu verstehen, ,wie verschieden die Kriege nach der Natur ihrer Motive und der Verhältnisse, aus denen sie hervorgehen, sein müssen“ (VK I/1/S. 212).

Ganz konkret bewegte die Frage nach dem historischen Wandel Clausewitz vor allem deswegen, weil er die „neuen Kriege“ seiner Zeit verstehen wollte, mit denen Napoleon Europa überzogen hatte (vgl. Herberg-Rothe 2001, Kap. 1). Im 18. Jahrhundert war der Krieg - so erzählt Clausewitz (VK VIII/3B/S. 967) die Geschichte - „ein bloßes Geschäft der Regierungen“, das diese mit stehenden Heeren und ohne größere Beteiligung des Volkes betrieben. Es ging nicht darum, den Gegner zu vernichten; vielmehr erschien der Krieg ,wie eine gegenseitige Entrüstung, um sich selbst zu schützen und dem anderen Furcht einzuflößen und gelegentlich ihm einen Streich beizubringen“ (VK VIII/2/S. 953). Das alles habe sich mit der Französischen Revolution und dann insbesondere mit den Napoleonischen Kriegen radikal geändert, denn der Krieg sei nun ,zur Sache des ganzen Volkes“ (VK VIII/3B/S. 972) geworden - erst auf der Seite Napoleons, dessen , auf die ganze Volkskraft gestützte Kriegsmacht zertrümmernd durch Europa [schritt]“ (VK VIII/3B/S. 972), dann auch auf Seite der europäischen Monarchien, die nun ebenfalls ihre ganzen Völker in die Waagschale warfen. Diese Transformation des Krieges galt es zu verstehen und die richtigen Konsequenzen daraus zu ziehen, wenn Preußen sich als europäische Macht behaupten wollte.

Für Clausewitz (VK VIII/6B/S. 997; vgl. VK VIII/3B/S. 972) haben die ,ungeheuren Wirkungen der französischen Revolution“ auf den Krieg vor allem in der „ganz veränderten Staats- und Verwaltungskunst, in dem Charakter der Regierung, in dem Zustande des Volkes“ ihren Ursprung, also in einem Wandel der politischen 
Verhältnisse. ${ }^{22}$ Dieser Wandel schlug sich zum einen in neuen, ambitionierteren politischen Zwecken nieder, das heißt, im Gehalt der Willen der Akteure. In den Kabinettskriegen des 18. Jahrhunderts ging es lediglich darum, ,,sich in einen mäßigen Vorteil zu setzen, um beim Friedensschluss davon Gebrauch zu machen“ (VK VIII/3B/S. 968). Mit der Französischen Revolution stand dann plötzlich nichts Geringeres auf dem Spiel als die politische und soziale Ordnung Europas. Deshalb ist Napoleon ,rastlos fortgeschritten, bis der Gegner niederlag; und fast ebenso rastlos sind die Rückschritte erfolgt" (VK VIII/2/S. 954). Zum anderen standen dem Feldherrn nun ganz andere Mittel der Gewaltanwendung zur Verfügung. Die Mobilisierung des gesamten Volkes in Massenaushebungen und durch die Volksbewaffnung erlaubte es den Regierenden, Krieg in einer zuvor undenkbaren Intensität zu führen (vgl. dazu Howard 1976, Kap. 5; Rink 2014). ${ }^{23}$ Mit Blick auf die Zukunft spekuliert Clausewitz, dass diese Mittel auch in allen nachfolgenden Konflikten, zumindest wenn die Zwecke von ausreichender Bedeutung sind, zur Anwendung kommen würden, denn es sei so, ,daß Schranken, die gewissermaßen nur in der Bewußtlosigkeit dessen, was möglich sei, lagen, wenn sie einmal eingerissen sind, sich nicht leicht wieder aufbauen lassen" (VK VIII/3B/S. 973). ${ }^{24}$ Dies ist freilich eine Vorhersage, die im weiteren Verlauf der Geschichte ihre grausame Bestätigung finden sollte.

Seit Napoleons letzter Schlacht bei Waterloo haben sich die Erscheinungsformen des Krieges radikal verändert. Clausewitz konnte nicht vorhersehen, wie genau neue Möglichkeiten der politischen Mobilisierung, die Mechanisierung der Kriegsführung, die Erfindung von Nuklearwaffen oder die Digitalisierung den Krieg verändern würden (vgl. Bousquet 2009; Howard 1976, Kap. 7, 8 und Nachwort). Mit jedem Schritt des politischen Wandels und mit jeder technischen Innovation haben freilich einige empirische Einsichten aus Clausewitz' Werk an Gültigkeit verloren. Und doch haben diese Veränderungen Clausewitz nicht widerlegt. Denn den eigentlichen Kern seines Denkens bildet gerade die Erkenntnis, dass die Erscheinungsformen der politischen Gewalt von den jeweiligen Verhältnissen ihrer Zeit geprägt werden. Solange die Politik als das Gegeneinanderhandeln von Akteuren verstanden werden kann, die Zwecke verfolgen, welche sie sich vor dem Hintergrund der Verhältnisse ihrer Zeit selbst gesetzt haben, und solange die Anwendung von Gewalt als Möglichkeit

\footnotetext{
${ }^{22}$ Hier folgt Clausewitz seinem Mentor Scharnhorst (1986, S. 97), der bereits 1797 beobachtet hatte, dass ,die Quelle des Unglücks, welches die verbundenen Mächte in dem französischen Revolutionskriege betroffen hat, [...] tief mit ihren inneren Verhältnissen und denen der französischen Nation verwebt sein [muss]“ (vgl. Münkler 2002b, S. 86; Wehler 1969, S. 228).

${ }^{23}$ Clausewitz schrieb am Ende einer Zeit, in der es kaum bedeutende Fortschritte in den Technologien des Krieges gegeben hatte, und so erschien ihm technischer Fortschritt auch nicht als eine starke Triebfeder des Wandels. „Von den neueren Erscheinungen im Gebiet der Kriegskunst“, beobachtet er, ,ist das allerwenigste neuen Erfindungen oder neuen Ideenrichtungen zuzuschreiben und das meiste den neuen gesellschaftlichen Zuständen und Verhältnissen“ (VK VI/30/S. 856).

${ }^{24}$ In Clausewitz' Analyse des Übergangs vom Kabinetts- zum Volkskrieg sind Zwecke und Mittel eng verwoben. Kriege können nur dann mit dem „ganzen Gewicht der Staaten“ geführt werden, wenn sie um „große, den Völkern naheliegende Interessen“ gehen (VK VIII/3B/S. 972). Dennoch hält er analytisch die Unterscheidung aufrecht, wenn er betont, ,daß das Ziel, welches sich der Kriegsunternehmer setzt, [und] die Mittel, welche er aufbietet [...] den Charakter der Zeit und der allgemeinen Verhältnisse in sich tragen werden“ (VK VIII/3B/S. 973).
} 
im Raum steht, solange kann die Politik auch in Clausewitz' Begriffen beschrieben werden.

Das gilt sicherlich für die „,neuen Kriege“ nach dem Ende des Ost-West-Konflikts, in denen neben Staaten, die im Übrigen immer noch wichtige Akteure sind, eben auch terroristische Netzwerke, Warlords, kriminelle Organisationen und manch eine private Firma versuchen, mit Gewalt ihren Willen gegen den Willen anderer Akteure durchzusetzen (Münkler 2002a, 2006; Wassermann 2015). ${ }^{25}$ Auch die jüngsten Erscheinungsformen des Krieges lassen sich mit Clausewitz deuten. So hat sich durch die langen Kriege in Afghanistan und Irak die US-amerikanische Militärdoktrin zwar insbesondere in Hinblick auf ihre Strategien und Techniken der Aufstandsbekämpfung verändert, doch zumindest in den Beschreibungen ihrer intellektuellen Architekt*innen operiert sie immer noch in der Clausewitz'schen Begriffswelt von politischem Zweck und militärischen Mitteln (Biddle 2014; Echevarria 2016). Auch Russlands „hybride“ Kriegsführung in der Ukraine und anderenorts scheint immer noch politische Zwecke und strategische Ziele zu verfolgen. Ihre Eigenheit ergibt sich vor allem ,,aus den eingesetzten Gewaltmitteln und ihrer kreativen Kombination“ (Wassermann 2016, S. 107; vgl. Galeotti 2016). Wenn man ,mit Clausewitz über Clausewitz hinaus“ (Münkler 2003, S. 7) denkt und seine Analyse des historischen Wandels des Krieges weiter schreibt, kann man also auch gegenwärtigen Formen der politischen Gewalt Bedeutung abgewinnen.

Die historische Betrachtung wirft aber auch noch einmal ein etwas anderes Licht auf das Verhältnis von Krieg und Gewalt. In der abstrakten Theorie ist es ja gerade der instrumentelle Gebrauch von Gewalt, der den Krieg von der übrigen Politik unterscheidet. In der Praxis lässt sich diese Binnengrenze innerhalb der Politik, die gewaltsames und gewaltloses Handeln, und somit Krieg und sonstige Politik, voneinander trennt, jedoch nicht so leicht ausmachen. Denn wenn der Krieg historisch verschiedene Grade der Intensität haben kann, die vom „Vernichtungskriege“ bis zur ,bewaffneten Beobachtung“ (VK I/1/S. 201) reichen, dann gilt es erst einmal zu klären, wann überhaupt die Gewalt vollständig abwesend ist und damit der Krieg endet. Clausewitz schreibt über den Kampf, also über die wechselseitige Anwendung von Gewalt: „Wie mannigfaltig dieser auch gestaltet sei, wie weit er sich von der rohen Erledigung des Hasses und der Feindschaft im Faustkampf entfernen möge, wieviel Dinge sich einschieben mögen, die nicht selbst Kampf sind, immer liegt es im Begriff des Krieges, daß alle in ihm erscheinenden Wirkungen ursprünglich vom Kampf ausgehen müssen“ (VKI/2/S. 222). Wenn die historisch tatsächlich stattfindenden Kriege auch oft mit so geringer Intensität geführt werden, dass eine echte Gewaltanwendung kaum stattfindet, so ist es doch die jederzeit herrschende Möglichkeit der Gewalt, die dem Krieg seine distinkte Form gibt. „Die Waffenentscheidung“ sei deshalb „für alle großen und kleinen Operationen des Krieges, was die bare Zahlung für den Wechselhandel ist“; denn ,wie entfernt diese Beziehungen auch seien, wie selten die Realisationen auch eintreten mögen, ganz können sie niemals fehlen“(VK I/2/S. 226).

\footnotetext{
25 Interessanterweise hat selbst Kaldor (2010) in einem jüngeren Aufsatz ihre Clausewitz-Interpretation revidiert und zugestanden, dass auch die neuen Kriege nach dem Ende des Ost-West-Konflikts mit rationalen Mitteln und für politische Zwecke geführt werden.
} 
Clausewitz hat nicht abschließend geklärt, wie weit entfernt die Möglichkeit einer Entscheidung mit den Waffen sein muss, damit Politik nicht mehr Krieg ist. In einer Zeit, in der Kriege gewöhnlich mit einer Kriegserklärung begannen und mit einem Friedenschluss endeten, stellte sich ihm diese Frage jedoch auch nicht mit großer Dringlichkeit. ${ }^{26}$ Ganz anders ging es den Bewundrer*innen des preußischen Generals im Kalten Krieg (z. B. Aron 1980; Brodie 1973; Howard 1981). Sie wollten mit Clausewitz' theoretischem Instrumentarium gerade einen Konflikt verstehen, in dem es - zumindest zwischen den Supermächten - kaum zu tatsächlicher Gewaltanwendung kam. ${ }^{27}$ Im nuklearen Zeitalter erschien die entscheidende Frage für die Clausewitzianer*innen gerade darin zu bestehen, wie lange sich durch die Drohung ihrer Anwendung der tatsächliche Einsatz von Atomwaffen aufschieben ließe. Oder in den bangen Worten Arons (1980, S. 455): „Kann man unendlich lange auf Kredit leben?" Wenn man das politische Wesen des Krieges ernst nimmt, schreibt Clausewitz (VK VIII/6A/S. 989), dann ,gibt es keine Grenze mehr“, und man muss auch solche Kriege in der Theorie berücksichtigen, ,die in bloßer Bedrohung des Gegners und in einem Subsidium des Unterhandelns bestehen“. Genau solch ein Krieg, in dem nicht gekämpft wird, sondern wechselseitige Drohungen die Grundlage von Verhandlungen bilden, ist der Kalte Krieg gewesen. ${ }^{28}$

Noch radikaler als die Strateg*innen des Kalten Krieges hat Foucault die Grenzen zwischen Krieg und Frieden verwischt, indem er Clausewitz' Begriff des Krieges auf alle Machtbeziehungen in modernen Gesellschaften ausgeweitet hat. ${ }^{29}$ Die Prämisse einer entsprechenden Theorie lautet für Foucault (2001, S. 32): „Macht ist Krieg, der mit anderen Mitteln fortgesetzte Krieg“. Die drei wichtigsten Implikationen fasst er folgendermaßen zusammen: Erstens wären die gesellschaftlichen Machtverhältnisse das Ergebnis vergangener Kriege und der Frieden wäre nichts als „ein stiller Krieg“, in dem diese Machtverhältnisse immer wieder aufs Neue ,in die Institutionen, die ökonomischen Ungleichheiten, in die Sprache und bis in die Körper" eingeschrieben werden (Foucault 2001, S. 32). Zweitens und folglich wären auch die Kämpfe um eine Veränderung der Machtverhältnisse in der Zivilgesellschaft nichts als eine „Fortsetzung“ oder auch „Episoden, Bruchstücke und Verlagerungen des Krieges“ (Foucault 2001, S. 33). Drittens kann auch in der bürgerlichen Gesellschaft „die letzte Entscheidung [...] nur vom Krieg gefällt werden, d.h. von einer Kraftprobe, bei der schließlich die Waffen entscheiden werden“" (Foucault 2001, S. 33). Für Fou-

\footnotetext{
26 Für Clausewitz selbst ist „Frieden“ allerdings kein zentraler Begriff. Wo er ihn verwendet, steht er für den Zustand nach dem Krieg, in dem die Akteure ihre Zwecke entweder erreicht oder verworfen haben (VK I/2/S. 215-17).

27 Ganz anders verhielt es sich allerdings im globalen Süden, in dem sich die Spannungen des Kalten Krieges in heißen Stellvertreterkriegen entluden; vgl. Westad (2005).

28 Durch die Erfindung der Nuklearwaffen haben sich jedoch die Vorzeichen radikal verkehrt. Während zu Clausewitz' Zeiten dann gedroht und nicht gekämpft wurde, wenn es um wenig ging, wird im Atomzeitalter nur gedroht und nicht gekämpft, weil alles auf dem Spiel steht; vgl. Aron (1980, S. 544).

29 Die Interpretation erschwert, dass Foucault glaubt, gegen Clausewitz zu argumentieren. Die Formulierung seiner eigenen Hypothese, der zufolge der tatsächliche Krieg als „Punkt maximaler Spannung“ (Foucault 2001, S. 62) einfach ein Extrem auf dem Kontinuum gesellschaftlicher Machtbeziehungen darstellt, kommt Clausewitz' Politikbegriff, so wie er in diesem Aufsatz von mir rekonstruiert wurde, dann aber sehr nahe; zu Foucaults Clausewitz-Rezeption vgl. Caygill (2013), Öberg (2014), Reid (2003).
} 
cault (2001, S. 62) kann man mit Clausewitz so „unterhalb von Frieden, Ordnung, Reichtum, Autorität, unterhalb der ruhigen Ordnung der Unterordnung, unterhalb des Staats und der Staatsapparate, unterhalb der Gesetze usw. eine Art primitiven Kriegs hören und wiederentdecken“. Der Krieg hört also niemals auf. Caygill (2013) hat mit seiner bereits erwähnten Theorie des Widerstandes genau an diese Überlegungen Foucaults zur verborgenen Gewalt der bürgerlichen Gesellschaft angeknüpft. Foucault und Caygill stehen somit für die radikale These, dass Clausewitz' Theorie der politischen Gewalt letztendlich eine adäquate Beschreibung aller Politik in der Moderne liefert.

Angesichts der Schwierigkeiten, innerhalb der historisch sich ereignenden Politik überhaupt einen Bereich ohne Gewalt auszumachen, werden dann auch Vorschläge verständlich, Clausewitz' berühmtes Diktum umzukehren. Aron (1980, S. 532), Foucault (2001, S. 32) und weitere Autor*innen (vgl. Guareschi 2010; Reid 2006) haben überlegt, was es heißen könnte, zu sagen, dass nicht der Krieg die Politik fortsetzt, sondern die Politik den Krieg. ${ }^{30}$ Betrachten wir Clausewitz' Begriffe systematisch in ihrem Bezug zueinander, ergibt so eine Aussage erst einmal wenig Sinn. Denn, wie wir gesehen haben, ist für Clausewitz jede denkbare kriegerische Handlung letztendlich politisch, aber nicht jede politische Handlung notwendigerweise kriegerisch. ${ }^{31}$ Der Krieg ist eine Unterkategorie der Politik. Anders sieht es jedoch aus, wenn wir Clausewitz' empirisch gesättigte Theorie vom Wandel des Krieges in der Geschichte betrachten. Denn wenn die Gewalt bei aller historisch stattfindenden Politik zumindest als Möglichkeit im Raum steht, dann ist die „Politik“ - nun allerdings verstanden als Periode, in der sich die Gewalt nicht wirklich materialisiert, in der man also, mit Arons Worten, auf Kredit lebt - tatsächlich eine Fortsetzung des Krieges, die vorübergehend auf einige seiner Mittel verzichtet. Nimmt man diese Perspektive ein, so erscheint jede sozialwissenschaftliche Forschung, die Politik ganz ohne den Krieg denkt, als höchst problematisch. Weil sie dazu beiträgt, die Spuren der Gewalt in der globalen Ordnung zu verbergen, ist sie selbst Ideologie (Barkawi 2016). Clausewitz' Theorie des Krieges erscheint hingegen als kritische Ressource, die dabei helfen kann, klarsichtig auf die Rolle von Gewalt in aller Politik zu blicken.

\section{Schlussbetrachtung: Sollten wir Clausewitz dennoch ,,vergessen“?}

In diesem Aufsatz habe ich Clausewitz' Theorie des Krieges als eine Theorie des Wandels der politischen Gewalt interpretiert. Dazu habe ich die zentralen Begriffe seines Denkens - „Politik“ und „Krieg“ - systematisch rekonstruiert. Politik ist in Clausewitz' allgemeiner Betrachtung das Gegeneinanderhandeln von zwei oder mehr Akteuren, die jeweils ihren eigenen Willen realisieren wollen, deren Wil-

\footnotetext{
30 Ebenfalls umgekehrt wurde das Diktum von militaristischen Autoren (z. B. Freyer 1925; Ludendorff 1935), die damit aber nicht vorrangig das Wesen des Krieges analytisch fassen, sondern viel mehr eine Unterordnung der zivilen Regierung unter das Militär rechtfertigen wollten (vgl. Wehler 1969).

31 Daase und Schindler (2009, S. 721) haben also erst einmal Recht, wenn sie schreiben: „Politik ist laut Clausewitz nicht die Fortsetzung des Krieges [...]. Krieg ist die Fortsetzung der Politik“.
} 
len sich jedoch wechselseitig in der Realisierung einschränken. Oftmals beziehen sich die Willen so aufeinander, dass der eine einen positiven Gehalt hat, also ein bestimmtes Vorhaben realisieren möchte, der andere aber insofern nur negativ bestimmt ist, dass er eben dieses Vorhaben vereiteln möchte. Der Krieg ist in diesem theoretischen Rahmen nichts als eine besondere Form der Politik, nämlich Politik, die mit den Mitteln der Gewalt betrieben wird. Aus dem besonderen Wesen der Gewaltmittel folgen jedoch gewisse Eigenheiten des Krieges, die ihn von der nichtgewaltsamen Politik unterscheiden, insbesondere eine Tendenz zur Eskalation und ein ermäßigendes Element der Reibung (das dort stärker ins Gewicht fällt als in der übrigen Politik). Eine Rolle in der Politik spielt die Gewalt jedoch nicht erst durch ihre Anwendung. Schon die Möglichkeit einer zukünftigen Anwendung von Gewalt kann ihren Schatten zurück auf die Politik werfen und sie so beeinflussen. Wie weit dieser Schatten reicht, ist letztendlich eine empirische Frage. Selbst wenn man sich nicht Foucaults radikaler Kritik des Liberalismus anschließen möchte, kann man jedoch sehen, dass die Dynamiken der politischen Gewalt, die Clausewitz theoretisch beschreibt, auch heute noch eine bedeutende Rolle in der globalen Politik spielen.

Schließen möchte ich meine Betrachtungen mit der Frage, ob wir uns von dem instrumentellen Verständnis der politischen Gewalt, das wir in Clausewitz' Denken über den Krieg finden, nicht trotzdem ein Stück weit lösen sollten. Die Schriften des preußischen Generals markieren einen historischen Wendepunkt, ab dem das Verhältnis zwischen Politik und Gewalt als eine Zweck-Mittel-Relation denkbar wurde. Gerade deswegen nimmt sein Diktum in Foucaults (2001, S. 58-81) genealogischer Untersuchung zum Wandel der politischen Macht einen so zentralen Platz ein. Wie eine Reihe von Studien zur Rezeptionsgeschichte seines Werkes (z. B. Heuser 2005; Kinross 2008) zeigen, hat Clausewitz' Verständnis des Krieges als Instrument der Politik seitdem nicht nur das politische und strategische Denken, sondern auch die politische und militärische Praxis stark geprägt. Sprechen wir heute über den Krieg, so wird oft als Erstes gefragt, was die Beteiligten politisch beabsichtigen und ob der Einsatz von Gewalt dafür zweckdienlich und verhältnismäßig ist. Doch, gerade weil die Vorstellung, Politik und Krieg seien untrennbar verbunden, so wirkmächtig ist, sollten wir auch fragen, welche Möglichkeiten im Denken und in der politischen Praxis durch sie verdeckt werden. Was können wir sehen und tun, wenn wir Clausewitz für einen Moment ,,vergessen“"?32

Zum einen wird der unpolitische und das heißt, nicht auf einen Zweck ausgerichtete, Krieg denkbar, wenn wir uns auf dieses Experiment einlassen und das die Moderne dominierende instrumentelle Verständnis der politischen Gewalt für einen Moment ausblenden. Genau diese Form des nichtpolitischen Krieges glauben van Creveld, Kaldor und andere Kritiker*innen Clausewitz' entdeckt oder auch wiederentdeckt zu haben. Nur sind die tatsächlichen Kriege unserer Tage, für die die Kritiker*innen dann eine neue Theorie fordern, wie wir gesehen haben, sehr wohl politisch in Clausewitz' Sinn. Die Akteure verfolgen zwar in diesen Kriegen nicht die Zwecke einer eng verstandenen Staatsräson; artikulierbare Zwecke verfolgen sie aber sehr wohl, seien diese nun ethnonational, religiös oder schlichtweg materi-

32 Genau das schlägt Öberg (2014) in einem Aufsatz mit dem Titel „Forget Clausewitz“ vor. Die Figur des Vergessens als Befreiung des Denkens nimmt er von Baudrillard (2007). 
ell. Falls es heute einen unpolitischen Krieg geben sollte, der sich mit Clausewitz' Theorie nicht fassen lässt, dann müsste dieser eine ganz andere, nämlich tatsächlich nicht auf einer Zweck-Mittel-Relation beruhende Form besitzen. Angesichts der Faszination, die die Idee eines nichtinstrumentellen, existenziellen Krieges auf viele Beobachter*innen ausübt, wird zudem leicht eine zweite Möglichkeit übersehen, die sich ebenfalls eröffnet, wenn wir uns von Clausewitz' Begriffen lösen: die gänzlich gewaltfreie Politik. Ob nun Politik jemals ganz, sogar als Ultima Ratio, auf Gewalt verzichten kann, ist freilich eine der großen Fragen der politischen Theorie (vgl. Arendt 1970; Sharp 1973). Klar ist aber, dass solch eine Politik in Clausewitz' Begriffen nicht zu fassen wäre. Wegen der engen Verknüpfung von Politik und Gewalt, die sie vornimmt, stößt Clausewitz' Theorie nämlich dort an ihre Grenzen, wo Politik ganz ohne Gewalt gedacht und praktiziert werden soll. Solange Gewalt faktisch ein Mittel ist, dessen sich Akteure in der Politik bedienen, ist diese Grenze allerdings keine Grenze der empirischen Anwendbarkeit seines Denkens; sie ist vielmehr eine Grenze unserer politischen Imagination.

Danksagung Mein Dank gilt Christopher Daase, Kristina Lepold, Sebastian Schindler und Philip Wallmeier sowie den zwei anonymen Gutachter*innen der PVS für ihre äußerst hilfreichen Kommentare und Anregungen zu verschiedenen Entwürfen dieses Aufsatzes. Des Weiteren wurde der Text im Dezember 2019 im Kolloquium der Hessische Stiftung Friedens- und Konfliktforschung diskutiert; ich danke den Anwesenden für ihre Fragen und Vorschläge. Thomas Siurkus sage ich Dank für seine Unterstützung bei der Fertigstellung des Manuskripts. Die Arbeit an diesem Aufsatz wurde von der Europäischen Kommission im Rahmen des Marie-Skłodowska-Curie-Projekts DIPLOWAR (Grant Agreement 792932) gefördert.

Förderung Die Europäische Kommission hat die Arbeit an diesem Aufsatz im Rahmen des Marie-Skłodowska-Curie-Projekts DIPLOWAR (Grant Agreement 792932) gefördert.

Funding Open Access funding provided by Projekt DEAL.

Open Access Dieser Artikel wird unter der Creative Commons Namensnennung 4.0 International Lizenz veröffentlicht, welche die Nutzung, Vervielfältigung, Bearbeitung, Verbreitung und Wiedergabe in jeglichem Medium und Format erlaubt, sofern Sie den/die ursprünglichen Autor(en) und die Quelle ordnungsgemäß nennen, einen Link zur Creative Commons Lizenz beifügen und angeben, ob Änderungen vorgenommen wurden.

Die in diesem Artikel enthaltenen Bilder und sonstiges Drittmaterial unterliegen ebenfalls der genannten Creative Commons Lizenz, sofern sich aus der Abbildungslegende nichts anderes ergibt. Sofern das betreffende Material nicht unter der genannten Creative Commons Lizenz steht und die betreffende Handlung nicht nach gesetzlichen Vorschriften erlaubt ist, ist für die oben aufgeführten Weiterverwendungen des Materials die Einwilligung des jeweiligen Rechteinhabers einzuholen.

Weitere Details zur Lizenz entnehmen Sie bitte der Lizenzinformation auf http://creativecommons.org/ licenses/by/4.0/deed.de. 


\section{Literatur}

Arendt, Hannah. 1970. Macht und Gewalt. München: Piper.

Aron, Raymond. 1980. Clausewitz: Den Krieg denken. Frankfurt am Main: Propyläen.

Barkawi, Tarak. 2016. Decolonising war. European Journal of International Security 1(2):199-214.

Bassford, Christopher. 1994. Clausewitz in English: the reception of Clausewitz in Britain and America, 1815-1945. New York: Oxford University Press.

Baudrillard, Jean. 2007. Forget Foucault. Los Angeles: Semiotext(e).

Beckmann, Rasmus. 2011. Clausewitz trifft Luhmann: Eine systemtheoretische Interpretation von Clausewitz' Handlungstheorie. Wiesbaden: VS Verlag.

Biddle, Stephen. 2014. Afghanistan's legacy: emerging lessons of an ongoing war. The Washington Quarterly 37(2):73-86.

Bousquet, Antoine J. 2009. The scientific way of warfare: order and chaos on the battlefields of modernity. New York: Columbia University Press.

Brodie, Bernard. 1973. War and politics. New York: Macmillan.

Brown, Michael E., Owen R. Coté, Sean M. Lynn-Jones, und Steven E. Miller (Hrsg.). 2004. Offense, defense, and war. Cambridge: MIT Press.

Caygill, Howard. 2013. On resistance: a philosophy of defiance. London: Bloomsbury.

von Clausewitz, Carl. 1966. Bekenntnisdenkschrift. In Carl von Clausewitz: Schriften, Aufsätze, Studien, Briefe, Bd. 1, Hrsg. Werner Hahlweg, 678-751. Göttingen: Vandenhoeck \& Ruprecht.

von Clausewitz, Carl. 1979. Gedanken zur Abwehr (Briefe vom 22. und 24.12.1827). In Carl von Clausewitz: Verstreute kleine Schriften, Hrsg. Werner Hahlweg, 495-530. Osnabrück: Biblio.

von Clausewitz, Carl. 1980. Vom Kriege, 19. Aufl., Hrsg. Werner Hahlweg. Bonn: Dümmler.

Collier, Paul, und Anke Hoeffler. 2004. Greed and grievance in civil war. Oxford Economic Papers 56(4):563-595.

van Creveld, Martin. 1997. What is wrong with Clausewitz? In The Clausewitzian dictum and the future of Western military strategy, Hrsg. Gert de Nooy, 7-24. Den Haag: Kluwer Law International.

van Creveld, Martin. 1998. Die Zukunft des Krieges. München: Gerling.

Daase, Christopher. 1999. Kleine Kriege - Große Wirkung: Wie unkonventionelle Kriegführung die internationale Politik verändert. Baden-Baden: Nomos.

Daase, Christopher. 2007. Clausewitz and small wars. In Clausewitz in the twenty-first century, Hrsg. Hew Strachan, Andreas Herberg-Rothe, 182-195. Oxford: Oxford University Press.

Daase, Christopher, und James W. Davis (Hrsg.). 2015. Clausewitz on small war. Oxford: Oxford University Press.

Daase, Christopher, und Sebastian Schindler. 2009. Clausewitz, Guerillakrieg und Terrorismus: Zur Aktualität einer missverstandenen Kriegstheorie. Politische Vierteljahresschrift 50(4):701-731.

Echevarria, Antulio J. 2007. On the Clausewitz of the Cold War: reconsidering the primacy of policy in "On war". Armed Forces \& Society 34(1):90-108.

Echevarria, Antulio J. 2016. Rediscovering US military strategy: a role for doctrine. Journal of Strategic Studies 39(2):231-245.

Fearon, James D., und David D. Laitin. 2003. Ethnicity, insurgency, and civil war. American Political Science Review 97(1):75-90.

Foucault, Michel. 2001. In Verteidigung der Gesellschaft: Vorlesungen am Collège de France (1975-76). Frankfurt am Main: Suhrkamp.

Freyer, Hans. 1925. Der Staat. Leipzig: Rechfelden.

Galeotti, Mark. 2016. Hybrid, ambiguous, and non-linear? How new is Russia's "new way of war"? Small Wars \& Insurgencies 27(2):282-301.

Gat, Azar. 1989. The origins of military thought: from the enlightenment to Clausewitz. Oxford: Clarendon Press.

Guareschi, Massimiliano. 2010. Reversing Clausewitz?: War and politics in Foucault, Deleuze-Guattari and Aron. In Conflict, security and the reshaping of society: the civilization of war, Hrsg. Alessandro Dal Lago, Salvatore Palidda, 70-83. London: Routledge.

Hahlweg, Werner. 1980a. Das Clausewitzbild einst und jetzt. In Carl von Clausewitz: Vom Kriege, 19. Aufl., Hrsg. Werner Hahlweg, 1-172. Bonn: Dümmler.

Hahlweg, Werner. 1980b. Die Weiterentwicklung und Differenzierung des Clausewitzbildes seit 1972. In Carl von Clausewitz: Vom Kriege, 19. Aufl., Hrsg. Werner Hahlweg, 1253-1340. Bonn: Dümmler.

Herberg-Rothe, Andreas. 2001. Das Rätsel Clausewitz: Politische Theorie des Krieges im Widerstreit. München: Fink. 
Herberg-Rothe, Andreas. 2007. Clausewitz's “wondrous trinity” as general theory of war and violent conflict. Theoria 54(114):48-73.

Herberg-Rothe, Andreas, und Jan Willem Honig. 2007. War without end(s): the end of Clausewitz? Distinktion: Journal of Social Theory 8(2):133-150.

Heuser, Beatrice. 2005. Clausewitz lesen!: Eine Einführung. München: Oldenbourg.

Heuser, Beatrice. 2010. Small wars in the age of Clausewitz: the watershed between partisan war and people's war. Journal of Strategic Studies 33(1):139-162.

Howard, Michael. 1976. War in European history. Oxford: Oxford University Press.

Howard, Michael. 1981. On fighting a nuclear war. International Security 5(4):3-17.

Howard, Michael. 1984. The influence of Clausewitz. In Carl von Clausewitz: On war, Hrsg. Michael Howard, Peter Paret, 27-44. Princeton: Princeton University Press.

Jäger, Thomas, und Rasmus Beckmann. 2011. Carl von Clausewitz' Theorie des Krieges. In Handbuch Kriegstheorien, Hrsg. Thomas Jäger, Rasmus Beckmann, 214-226. Wiesbaden: VS Verlag.

Kaldor, Mary. 2000. Neue und alte Kriege: Organisierte Gewalt im Zeitalter der Globalisierung. Frankfurt am Main: Suhrkamp.

Kaldor, Mary. 2010. Inconclusive wars: is Clausewitz still relevant in these global times? Global Policy 1(3):271-281.

Keegan, John. 1997. Die Kultur des Krieges. Reinbek: Rowohlt.

Keen, David. 1998. The economic functions of violence in civil wars. Oxford: Oxford University Press.

Kessel, Eberhard. 1987. Zur Genesis der modernen Kriegslehre. Die Entstehungsgeschichte von Clausewitz' Buch Vom Kriege. In Eberhard Kessel: Militärgeschichte und Kriegstheorie in neuerer Zeit Ausgewählte Aufsätze, Hrsg. Johannes Kunisch, 122-147. Berlin: Duncker \& Humblot.

Kinross, Stuart. 2008. Clausewitz and America: strategic thought and practice from Vietnam to Iraq. London: Routledge.

Levy, Jack S. 1984. The offensive/defensive balance of military technology: a theoretical and historical analysis. International Studies Quarterly 28(2):219-238.

Ludendorff, Erich. 1935. Der totale Krieg. München: Ludendorffs Verlag.

Münkler, Herfried. 2002a. Die neuen Kriege. Reinbek: Rowohlt.

Münkler, Herfried. 2002b. Über den Krieg: Stationen der Kriegsgeschichte im Spiegel ihrer theoretischen Reflexion. Weilerswist: Velbrück Wissenschaft.

Münkler, Herfried. 2003. Clausewitz’ Theorie des Krieges. Baden-Baden: Nomos.

Münkler, Herfried. 2006. Der Wandel des Krieges: Von der Symmetrie zur Asymmetrie. Weilerswist: Velbrück Wissenschaft.

Münkler, Herfried. 2007. Clausewitz and the privatization of war. In Clausewitz in the twenty-first century, Hrsg. Hew Strachan, Andreas Herberg-Rothe, 219-230. Oxford: Oxford University Press.

Öberg, Dan. 2014. Forget Clausewitz. International Journal of Baudrillard Studies 11(2). http:// baudrillardstudies.ubishops.ca/forget-clausewitz/.

Osgood, Robert E. 1957. Limited war: the challenge to American strategy. Chicago: University of Chicago Press.

Paret, Peter. 1992. Understanding war: essays on Clausewitz and the history of military power. Princeton: Princeton University Press.

Reid, Julian. 2003. Foucault on Clausewitz: conceptualizing the relationship between war and power. $A l$ ternatives: Global, Local, Political 28(1):1-28.

Reid, Julian. 2006. Re-appropriating Clausewitz: the neglected dimensions of counter-strategic thought. In Classical theory in International Relations, Hrsg. Beate Jahn, 277-295. Cambridge: Cambridge University Press.

Rink, Martin. 2014. The German wars of liberation 1807-1815: the restrained insurgency. Small Wars \& Insurgencies 25(4):828-842.

Rosinski, Herbert. 1935. Die Entwicklung von Clausewitz’ Werk ,,Vom Kriege“ im Lichte seiner ,Vorreden“ und „Nachrichten“. Historische Zeitschrift 151(2):278-293.

von Scharnhorst, Gerhard Johann David. 1986. Entwicklung der allgemeinen Ursachen des Glücks der Franzosen in dem Revolutionskriege und insbesondere in dem Feldzuge von 1794. In Scharnhorst: Ausgewählte militärische Schriften, Hrsg. Hansjürgen Usczeck, Christa Gudzent, 97-150. Berlin: Militärverlag der Deutschen Demokratischen Republik.

Scheipers, Sibylle. 2017. "The most beautiful of wars": Carl von Clausewitz and small wars. European Journal of International Security 2(1):47-63.

Scheipers, Sibylle. 2018. On small war: Carl von Clausewitz and people's war. New York: Oxford University Press.

Schindler, Sebastian. 2020. Clausewitz zur Einführung. Hamburg: Junius. 
Schuurman, Bart. 2010. Clausewitz and the "new wars" scholars. Parameters 40(1):89-100.

Sharp, Gene. 1973. Power and struggle. Boston: Porter Sargent.

Sumida, Jon Tetsuro. 2008. Decoding Clausewitz: a new approach to On war. Lawrence: University Press of Kansas.

Summers, Harry G. 1984. On strategy: a critical analysis of the Vietnam war. New York: Dell.

Villacres, Edward J., und Christopher Bassford. 1995. Reclaiming the Clausewitzian trinity. Parameters 25(3):9-19.

Vollrath, Ernst. 1984. „Neue Wege der Klugheit“: Zum methodischen Prinzip der Theorie des Handelns bei Clausewitz. Zeitschrift für Politik 31(1):53-76.

Wassermann, Felix. 2015. Asymmetrische Kriege: Eine politiktheoretische Untersuchung zur Kriegführung im 21. Jahrhundert. Frankfurt am Main: Campus.

Wassermann, Felix. 2016. Chimäre statt Chamäleon: Probleme der begrifflichen Zähmung des hybriden Krieges. Sicherheit \& Frieden 34(2):104-108.

Wehler, Hans-Ulrich. 1969. „Absoluter“ und „totaler“ Krieg: Von Clausewitz zu Ludendorff. Politische Vierteljahresschrift 10(2/3):220-248.

Westad, Odd Arne. 2005. The global Cold War: Third World interventions and the making of our times. Cambridge: Cambridge University Press. 